\title{
Underwater Ad-Hoc Networks: A Review
}

This paper was downloaded from TechRxiv (https://www.techrxiv.org).

\section{LICENSE}

CC BY-SA 4.0

SUBMISSION DATE / POSTED DATE

$12-11-2021 / 17-11-2021$

CITATION

Wengle, Emil; Potter, John; Dong, Hefeng (2021): Underwater Ad-Hoc Networks: A Review. TechRxiv. Preprint. https://doi.org/10.36227/techrxiv.17000266.v1

$\mathrm{DOI}$ 


\author{
Emil Wengle, John Potter, Senior Member, IEEE, \\ and Hefeng Dong, Member, IEEE
}

\begin{abstract}
Underwater sensor networks have become increasingly interesting in the past four decades. They can be used in a multitude of scenarios, commercial and military alike. Underwater networks can communicate in several ways, but when nodes are far apart, underwater acoustic communication is the only feasible way. The complex underwater acoustic channel puts high demands on the network protocols. The physical layer needs to contend with short coherence times, high intersymbol interference and significant Doppler spread. The routing protocol needs to handle intermittent connectivity and mobile network topologies, such as autonomous underwater vehicle networks. The medium access control protocol needs to manage medium access with high latency and potentially high packet loss ratios without congesting the network. The available acoustic modems are still rather expensive, which limits the size of a sensor network. Voices have also been raised from the academia for a paradigm shift, from hardware-defined, proprietary modems to software-defined, open-architecture modems, in order to accelerate research in the field and enable interoperability. This paper reviews the recent advancements in designing and implementing underwater networks on several levels and discusses some interesting approaches to underwater ad-hoc networking. The focus lies on acoustic communication.
\end{abstract}

Underwater acoustic communication, underwater networks, underwater ad-hoc networks.

The authors are with the Acoustics Group, Department of Electronic Systems, Norwegian University of Science and Technology, NO-7491 Trondheim, Norway.

Manuscript received November 12, 2021.

\title{
I. Introduction
}

Underwater acoustic communication has seen an increase in demand in the last four decades. There are many applications of underwater acoustic networks, including, but not limited to, wave power site monitoring [1], natural disaster warning systems, sea bottom mapping and 
nomena make underwater acoustic communication much more difficult than terrestrial radio frequency (RF) communication. Thus, early acoustic communication relied on incoherent modulation schemes or differential schemes. It was robust, but the achieveable symbol rate-range product was low [9]. More recent networks can use coherent modulation schemes thanks to more advanced receiver structures and the development of decision feedback equalisers (DFEs) and phase-locked loops (PLLs). However, they still have to contend with the difficult time-varying channel.

In this article, we review the state of the art in underwater ad-hoc networking. A wireless adhoc network is a network formed by wireless nodes without intervention from a base station or similar network master [10]. An example of such a network is an AUV network. While there is plenty of research on terrestrial ad-hoc wireless networks, most of the research on underwater adhoc wireless networks has come in the last decade. Our article focuses on acoustic communication and networking.

The remainder of this article is structured as follows. Section II discusses the underwater acoustic channel, motivates the need for underwater acoustic communication, presents some channel models and discusses standardisation. Section III discusses acoustic modems, how they are designed, transducer design and some software-defined approaches. Section IV discusses the physical layer, challenges that are best handled at the physical layer, and others' efforts in the physical layer. Section V discusses medium access control (MAC), which is an important feature in the data link layer, challenges in underwater MAC and others' approaches to MAC. Section VI discusses routing, which is an important feature in the network layer, along with challenges in routing in underwater networks and what others have done within routing. Section VII discusses some approaches to networking and some notable perspectives of underwater networks. Section VIII concludes the paper.

\section{Underwater acoustic channel}

The acoustic channel in one geographical location can be completely different to the channel in another location, and there is no way to define a "typical" underwater acoustic channel, nor is there any widely accepted set of canonical acoustic channels or channel simulators. [11] studies the acoustic channel in several waters in northern Europe, including the continental shelf, the Baltic sea, and Norwegian fjords. It was found that the channel can be overspread, which means the product of its delay spread and Doppler spread is large, or reverberant; stationary, 

does not need.

\section{B. Challenges} channel. [7] notes six challenges with designing an underwater network, some of which are

Terrestrial networks use electromagnetic (EM) waves to relay information to other nodes. This works because air is an insulating medium. Water is a conductive medium, and so it absorbs RF waves. In fact, EM waves must be sent at frequencies down to $30 \mathrm{~Hz}$ to propagate any significant distance through water [7]. To make matters worse, this would require a prohibitively big antenna and cause high power consumption, which is infeasible.

Underwater networks can use optical communication. It has some advantages over acoustic communication. First, light propagates at over $2.2 \times 10^{8} \mathrm{~m} \mathrm{~s}^{-1}$ in water, compared to $1500 \mathrm{~m} \mathrm{~s}^{-1}$ of sound. Second, visible light has extremely high frequency, so the achieveable data rates are limited by other factors. Third, optical communication offers higher bandwidth than acoustic communication thanks to the higher carrier frequency that optical communication uses.

Still, there are situations where acoustic communication is preferred over optical communication. First, water absorbs light; while not as strongly as RF waves, it means the range is confined to tens of metres [15]. Using acoustic waves is feasible even at distances on the order of kilometres given an appropriate frequency band, which makes acoustics a popular choice for sensor networks. Second, optical communication works best with line of sight, which acoustics

The underwater acoustic channel is much more complicated than the wireless terrestrial 
due to the nature of the channel.

- The acoustic waves propagate very slowly, compared to radio waves in air. This means latency will be extremely high. It also varies significantly over time.

- The acoustic waves can travel from the source to the receiver in multiple ways due to surface or bottom reflection as well as the sound speed profile. This is known as multipath propagation. This, along with fading, impairs the channel greatly.

- The bandwidth available to the network is very limited.

- Multipath propagation impairs the channel, and the underwater channel contains shadow zones, where the transmission loss is even stronger. Their location depends on range, the transmitter depth, the sound speed profile and bathymetry and may vary over time.

- Most sensors are battery-powered, and there is typically no way for them to recharge their batteries. This raises the need for low-power solutions in underwater sensor networks, especially in long-lived networks.

- The underwater environment is extreme. Sensors are constantly exposed to corrosion and other types of degradation.

Efforts have been made to overcome these challenges. [16] presents the design of an energyharvesting sensor. It extracts energy from ultrasonic waves and stores it in a supercapacitor. This energy can later be expended by the node itself to transmit. The underwater sensor network could thus last longer with energy harvesting.

\section{Channel model}

There exists a variety of models related to the underwater acoustic channel. One of the first models is the Urick transmission loss model [17], which is an empirical model of how transmission loss increases with range and carrier frequency. Thorp proposed an approximate formula for the absorption coefficient for low frequencies [18]. It states that absorption loss increases with frequency. The Urick model is easy to implement in a resource-limited environment, such as on a microcontroller, but it does not capture the effects of multipath propagation. The ray-tracing software Bellhop [19] provides a more accurate picture of the transmission loss in space, but requires substantial resources to run.

[20] presents a statistical channel model that includes both small-scale and large-scale effects. 
From the Urick model, each path acts as a nonlinear low-pass filter with gain

$$
\bar{H}_{p}(f)=\frac{\Gamma_{p}}{\sqrt{A\left(\bar{l}_{p}, f\right)}},
$$

where $\bar{H}_{p}$ is the path gain, $\Gamma_{p}$ is the combined effect on amplitude and phase of all reflections against the surface and sea bottom, $A$ is the Urick transmission loss, and $\bar{l}_{p}$ is the path length. The transfer function is then separated into $\bar{H}_{0}(f)$ and $h_{p}$. [20, fig. 1] shows the reference $\bar{H}_{0}(f)$ for path length $\bar{l}_{0}=1 \mathrm{~km}$ and spherical spreading $(k=2) . \bar{h}_{p}=\Gamma_{p}\left(\left(\bar{l}_{p} / \bar{l}_{0}\right)^{k} a_{0}^{\bar{l}_{p}-\bar{l}_{0}}\right)^{-1 / 2}$ is the path-specific gain relative to $\bar{H}_{0}$. Combining the transfer functions of each path gives the nominal channel transfer function

$$
\bar{H}(f)=\bar{H}_{0}(f) \sum_{p=1}^{N} \bar{h}_{p} e^{-\mathrm{j} 2 \pi f \bar{\tau}_{p}},
$$

where $\bar{\tau}_{p}$ is the nominal propagation delay relative to a reference delay $\tau_{0}$ and $\mathrm{j}$ is the imaginary unit. The path gain follows a log-normal distribution, provided the deviation in path length is Gaussian.

The path gain captures the large-scale effects well, but not the small-scale effects. [20] treats them on a per-path basis. Scattering occurs when a ray strikes a rough surface or a small object and splits into several smaller rays. Each smaller ray has a fading coefficient $\gamma_{p, i}$ and a phase distortion that depends on $\delta \tau_{p, i}$. Summing over each micropath gives a cumulative fading coefficient

$$
\gamma_{p}=\sum_{i} \gamma_{p, i} e^{-\mathrm{j} 2 \pi f \delta \tau_{p, i}}
$$

whose distribution converges to a complex Gaussian by the central limit theorem. The $\gamma_{p, i}$ can be assumed to be independent of each other. It is conditioned on both large-scale effects (delay and gain) and the mean and variance, and it may also vary over time by an autoregressive stochastic process. The delay can also be modelled on state space form, where the noise term is a complex Gaussian process. The resulting distribution becomes statistically equivalent to the previously mentioned one.

[20] also treats Doppler shift as a small-scale effect, which affects the exponent in (2). It varies slowly, so it can be considered piecewise constant. There are several sources of Doppler shift, including, but not limited to, surface motion, drifting, and intended motion. These sources can be assumed independent of each other, so the autocorrelation function, which can be used to characterise Doppler shift, becomes a product of expected values. Any factor that is deemed deterministic will not contribute to the autocorrelation. This autocorrelation becomes a product of 
Bessel functions of the first kind and order zero; see [20, §4] for details. All of these phenomena combined results in the "complete" channel model [20]

$$
H(f, t)=\bar{H}_{0} \sum_{p} h_{p} \bar{\gamma}_{p}(f, t) e^{\mathrm{j} 2 \pi f \tau_{p}},
$$

where the quotation marks stress that not all small-scale phenomena are included in the model.

\section{Shallow and deep channels}

The underwater acoustic channel is treated differently depending on its depth. Shallow channels are typically a few hundred metres deep at most, and deep channels are deeper than that.

The shallow channel typically experiences multipath propagation due to surface and sea bottom interaction more than refraction, particularly at shorter range. The sound speed profile can in some cases be considered constant. Multipath in the deep channel is more often caused by signal refraction due to the nonhomogeneous sound speed profile. This normally creates convergence zones, where the signal is stronger, and shadow zones, where the signal is remarkably weaker.

[21] considers the shallow harbour environment and how the stronger reverberation affects communication performance. The channel was measured by transmitting trains of variablerate linear frequency modulation (LFM) probe signals over a wide frequency band. Shorter pulses helps with estimating small-scale variability, and longer pulses helps with capturing late arrivals. Two experiments were analysed: one at $100 \mathrm{~m}$ range, one at $2.5 \mathrm{~km}$ range. Somewhat surprisingly, the results indicate that short-range communication is more difficult than long-range communication. Structures in the harbour do not appear to affect signals remarkably, unless they are near either node that is involved in communication.

\section{E. Standardisation}

Despite the fact that there are a handful of manufacturers of acoustic modems available on the market [22]-[25], there has been little development on standards for underwater acoustic communication. There are no canonical underwater acoustic channels or underwater network simulators available, although there exist research efforts to establish them [26]. Researchers that have designed their own algorithms for underwater networking have often had to write their own simulators or extensions of existing simulators, like the discrete-event network simulator ns-2, as a consequence. The limitations of their simulators are often not given in their work, which means it is difficult to determine their validity [27]. 
Due to the absence of underwater acoustic communication standards, most modem manufacturers develop their own protocols, architecture and system. This is a major obstacle for manufacturer interoperability and also communication efficiency [28].

To the best of our knowledge, the only standard that exists to this day is Janus, which was developed by NATO [29]. It is robust and easy to implement by design. Janus uses frequencyhopping binary frequency shift keying (FHBFSK) with 13 frequency pairs that together span a frequency band about one third of the centre frequency wide. It also employs convolutional coding at 1/2 data rate, interleaving and 8-bit cyclic redundancy check (CRC). Also, Janus has a detailed packet specification. It uses carrier sense multiple access (CSMA) with exponential backoff for medium access control. [29] defines Janus only mathematically, but there exist implementations of the protocol online [30]. Extensions to and derivative works of Janus have been developed [31].

[32] compares the performance of Janus against an frequency-repetition spread spectrum (FRSS)-based physical layer protocol. The conclusion made from the results is that Janus may become the weakest link in the SALSA stack without enhancements to the Janus receiver.

[28] presents four services that could use Janus. The first is making first contact and switching language, where Janus would serve as a common language, or lingua franca, to enable modems from different manufacturers to communicate. The second is an underwater automatic identification system, which can be used to help prevent accidents between underwater assets, such as submarines, and surface vessels. The third is underwater meteorology and oceanography, which is about forecasting the underwater environment. The forecasting data can be used by AUVs to adapt and make decisions about their current mission. Janus has been an attractive means of communication in search and rescue operations, so the fourth case is thus supporting distressed submarine operations. In conclusion, Janus can supersede or complement the underwater telephone.

\section{Acoustic modems}

Digital underwater acoustic communication is made possible by acoustic modems. An acoustic modem consists of a transducer for sending and receiving acoustic signals, an analogue transceiver that preprocesses signals for transmission and received signals, signal converters and a digital hardware platform that performs signal processing and control [33]. 
There exist many different underwater acoustic modems and manufacturers. [34] offers an exhaustive study on existing modems, reviewing their hardware and comparing research modems to commercial modems. The significant difference between research and commercial is that commercial modems generally achieve higher bit rates at the cost of higher power consumption, while research modems are more energy-efficient and better suited for deployment in sensor networks. There is still a need for more energy-efficient modems, however.

Terrestrial RF modems are available for consumers and can typically be bought at any electronics store for less than 100 euros today. Buying acoustic modems, however, is very difficult ${ }^{1}$ if the buyer is not a company in academy or industry, as one modem typically costs thousands of euros. This makes them monetarily intractable for sensor networks.

Research efforts have been made to design low-cost modems; for instance, [33] presents an acoustic modem that would cost 600 US dollars (2010, estimated) per unit if constructed in bulk. The modem had not been tested in the ocean because not all components had been developed at that time. On the other hand, [35] presents their "nanomodem" that costs only $£ 50$ per unit to manufacture. This modem has been tested in sea trials in conjunction with a MAC protocol that is proposed in [36].

\section{A. Transducer design}

The transducer is perhaps the most important component in an acoustic modem, as it is responsible for producing sound. According to the authors of [33], the transducer is also the most expensive component.

The transducer is usually omnidirectional, which means it produces sound waves in all directions. This is problematic for full-duplex modems, as it causes self-interference. [37] proposes a beamforming method to reduce this self-interference. The transducer used is a phased array transducer, which in this scenario is a $12 \times 12$ array of piëzoelectric ceramic elements. A subset of them is used for beamforming, and the beam can be rotated electronically to a certain extent, as overdoing it causes grating lobes to appear. The range of suitable angles depends on the frequency used and the element spacing.

\footnotetext{
${ }^{1}$ The buying process often involves requesting a quote, and the process from start to finish could easily take months.
} 


\section{B. Software-defined modems}

The early commercial off-the-shelf acoustic modems have a proprietary architecture, which often is hardware-based and rigid in its design. They rarely allow any reconfiguration, and when they do, the available settings are few. This makes them unfit for adaptive solutions to underwater networking and inhibits research efforts like IoUT. Consequently, groups of researchers have argued for the need for a paradigm shift towards software-defined architecture [27], [38]-[40].

[41] conducts a review of the literature on software-defined modems and academic and industrial advancements in this field. The early modems were black-box, as the physical layer was firmware-defined and the network protocol held fixed. Later modems supported reconfiguration, letting the user adjust coding scheme and bit rate on the fly. There were also reprogrammable modems. Those modems allowed the user to redefine one or more layers in the network stack, but this would often mean programming a digital signal processor (DSP) or field programmable gate array (FPGA), or programming in a specialised language, unless the modem is softwaredefined. Open-source and open-architecture are two features that facilitate accessibility. The authors list and classify a multitude of available modems from four aspects: physical-layer reprogrammability, network-layer reprogrammability, architecture availability and architecture generalisation. They also present the NILUS node, whose modem is explicitly designed to support operation on a general-purpose processor and be independent of specialised hardware.

[38] presents a real-time adaptive software-defined modem. Most of the adaptive functionality is implemented in the physical layer. Commercially available modems normally achieve data rates short of $35 \mathrm{kbit} / \mathrm{s}$, but as mentioned in Section IV-B, their modem achieves $260 \mathrm{kbit} / \mathrm{s}$ under certain conditions.

[39] proposes a software-defined architecture called SoftWater, which uses network virtualisation concepts to incorporate energy-efficient and robust underwater communication solutions. SoftWater supports acoustic communication, but also optical communication and magnetic induction-based communication. Programmability, cooperativeness, openness and virtualisability are the four core features of SoftWater. Network function virtualisation leads to a flexible architecture and facilitates network management and control in a centralised network topology. Network virtualisation lets isolated virtual networks share physical network infrastructure without interfering with each other.

[40] introduces the SEANet project as a step towards enabling IoUT on an open architecture 
and a software-defined platform. There is the need for a modem that can sacrifice acoustic range in exchange for data rate and a transducer that can operate at a wide range of frequencies. The prototype uses two transducers: one commercial off-the-shelf transducer for communication at up to $170 \mathrm{kHz}$ and one custom-made microelectromechanical systems (MEMS) piëzoelectric micromachined transducer array for communication about $700 \mathrm{kHz}$ with a bandwidth of $500 \mathrm{kHz}$. The modem can achieve data rates near $600 \mathrm{kbit} / \mathrm{s}$ at bit error rate (BER) not exceeding 0.01 and signal to noise ratio (SNR) at least $10 \mathrm{~dB}$.

[42] outlines a software-defined open-architecture modem, where the architecture is based on a high-level OSI generalisation. Each layer is represented by a module with a policy engine providing layer functionality. A cross-layer framework allows additional information to be aggregated in the data packets.

\section{Physical layer}

The physical layer is the lowest layer in the OSI stack model, and interacts directly with the transmitter and receiver. This layer supports data coding, modulation, demodulation and decoding as well as signal detection, to mention some of its important features [43]. This section discusses physical layer features and the challenges that the physical layer faces in the field of underwater networking.

\section{A. Challenges in the physical layer}

As mentioned in Section II-C, underwater sensor nodes are typically battery-powered and cannot recharge. Energy efficiency is therefore important, especially if the network should operate for weeks or longer periods of time.

Unless the channel is particularly benign, it will distort the phase of acoustic signals significantly. This puts great demands on the receiver side of the physical layer if signals are to be modulated in phase domain, such as by phase shift keying (PSK) or quadrature amplitude modulation (QAM). Frequency shift keying (FSK) is easier on the receiver side, but it uses more bandwidth than other modulation schemes [7].

\section{B. Approaches to the physical layer}

In [38], a software-defined modem with real-time adaptivity in the physical layer is presented. The adaptive physical layer is based on an optimisation problem, with the modulation order and 

filter taps as if the channel was a discrete-time noncausal linear, time-varying filter.

[26] presents a Matlab-based benchmarking software called Watermark. The purpose of Watermark is to provide a standard test bench such that physical-layer algorithms can be tested in a standardised way. It uses channel estimates that are derived from measured impulse responses and relies on libraries with measured underwater channels from different geographical sites.

[47] presents an experiment that took place in an area in the Grevelingen estuary, the Netherlands. The channel estimates were obtained by probing. Also, the nodes used two different frequency bands, where each band had specific physical layer functionality. Using two different frequency bands allows a node to serve as a potential bridge between two networks 
(ISI) and estimate the symbols with ISI, and a feedback filter that estimates the ISI from a sequence of recent symbols and eliminates it. The adaptive receiver is shown by experiment to successfully detect QPSK and 8PSK symbols in both deep and shallow water, even when the input symbols are smeared beyond recognition by inspection.

\section{Medium access control}

Medium access control, or MAC for short, is a core feature of the data link layer. It governs how the users in a network can use the available spectrum and ensures that the sent packets are successfully received, whether the users employ multiple access or random access [10]. Multiple access splits a channel in one of its dimensions, such as time, frequency or coding, into subchannels that different users can be assigned. It also involves control mechanisms for assigning channels and refusing users that do not fit in the system. Random access is a MAC strategy where users are assigned a channel dynamically. Unlike multiple access, assigning channels and refusing users to transmit is embedded in the protocol.

\section{A. Challenges in MAC}

As with many other network features, the challenging underwater acoustic channel makes MAC more difficult in underwater acoustic networks. [7], [53], among others, say that not all of the traditional multiple or random access protocols work well underwater. Frequency division multiple access (FDMA) does not work well due to multipath and frequency-selective fading. Time division multiple access (TDMA) typically does not work well due to propagation delay. The slow propagation speed is usually an inconvenience, as it makes contention-based MAC difficult to implement. However, [54] showed that the long propagation delay can be exploited to drastically increase potential network throughput. CSMA helps preventing packet collisions at the transmitting side, but it needs guard time to prevent receiver-side collisions. Code division multiple access (CDMA) is a more promising MAC technique, as it lets users exploit time diversity by Rake filters.

\section{B. Approaches to MAC}

TDMA, as discussed in Section V-A, does not work very well underwater due to the long guard times necessary, unless propagation delay is exploited. [54] proposes a schedule-based TDMA strategy, where nodes can either transmit, receive or be idle in one time slot. Packets may collide 
with each other as they traverse the channel. The simplest case considers networks where the distance between all pairs of nodes is an integer, and it can be extended to rational distances. It is shown that the upper bound on the throughput of the delay-exploiting TDMA strategy equals half the number of nodes in the network. An algorithm for finding near-optimal schedules in polynomial time for a given network topology is also presented. The reverse problem, finding a suitable network topology for a given schedule, is also considered. This puts strict requirements on clock synchronisation between the nodes, for clock drift could easily ruin a carefully designed schedule.

[55] proposes DACAP, a distance-aware collision avoidance protocol, for ad-hoc underwater acoustic networks with long delay. It lets nodes adjust the handshake length to the intended receiver to reduce average handshake duration. The protocol is request to send (RTS)/clear to send (CTS)-based, and nodes that have CTS listen for warning packets or other packets before transmitting, entering a back-off state if any such packets are overheard. Incoming RTS packets are treated separately. Acknowledgement packets are optional.

[56] analyses the MAC strategies frequency-hopping spread spectrum (FHSS) and DSSS and presents two direct-sequence receivers. FHSS divides the available band in bins and hops between them in a minimal-interference way, and DSSS uses codes with suitable correlation to spread the PSK-modulated data. The DSSS receivers are tested with FHSS and DSSS signals in shallow water and at short range. FHSS is very simple to implement, but has higher BER than DSSS at the same SNR.

[57] discusses the Stop and Wait protocol type. When the transmitter has sent a packet, it waits for an acknowledgement for a set duration. Unless it receives a positive one, it sends the packet again, and on receiving a positive acknowledgement, the transmitting node moves on to the next packet. This works in half-duplex systems, but the efficiency degrades remarkably with latency. Solutions exist where several packets are sent at once to make better use of the channel, and they achieve higher throughput efficiency than the single-packet S\&W. Throughput efficiency is a measure of how well the channel is exploited and is defined as the ratio of useful packet time to expected total packet transmission time.

[58] proposes a distributed MAC protocol that builds on lightweight and distributed noncooperative optimisation algorithms. Each transmitter optimises a local transmission probability profile from past observations of interference. It can proactively adapt its transmission strategy to keep packet losses down to a minimum, but also keep transmission probability high as to avoid 
unnecessarily long packet delay. The optimisation problem that the nodes solve is quasiconvex. Also, it has room for enhancement by integrating CDMA or FDMA.

\section{Routing}

Routing is an important feature in the network layer. It is used in networks where transmitting data between two distant nodes is too difficult or would require too much power. Rather than sending data to a destination directly, the source node can use intermediate nodes as data relays, although there exist techniques that make better use of multiuser diversity [10].

Routing protocols can generally be divided in three classes [7], [17], [53]. Proactive protocols focus on establishing routes as soon as the network is enabled and when its topology changes. This approach does not scale well and is not always necessary in underwater networks. Reactive protocols focus on on-demand route discovery. While it does not require activation time, it does require a wave of control packets for route building. Also, static network topologies are unlikely to need routing in this way. Geographical protocols require positional information, but typically scale better than proactive and reactive protocols. The long propagation delay underwater can be exploited to localise other nodes and establish routes.

\section{A. Challenges in routing}

Routing is a considerable challenge in ad-hoc wireless networks, especially when nodes are nonstationary. A route that works well at one occasion may not work at all at the next occasion. There is thus the need for dynamically reconfiguring routing protocols in ad-hoc networks [10]. There are several types of underwater networks. Sensor networks could span several kilometres in range, and fully mobile networks (of AUVs/ROVs) may experience rapidly varying channels. As such, all networks cannot rely on a universal routing protocol and always expect good performance.

[7] formulates eight research challenges in the network layer. Some of them are beyond the scope of this article and are not listed here.

- Delay-sensitive applications need algorithms that impose bounds on data latency.

- Routing protocols that handle unexpected disconnections in a local context should be developed.

- Consistent variations in energy efficiency metrics need local route optimisation algorithms to handle them. 
- AUV-to-sensor communication and AUV integration in networks needs special mechanisms.

\section{B. Approaches to routing}

A common approach to routing in ad-hoc networks is to use a distributed strategy for routing. Nodes broadcast information about their connectivity to nearby nodes, then the nodes compute new routes from it. This reduces routing overhead and is easy to adapt to changes in connectivity and links, but it is normally suboptimal and may result in routing loops [10].

The type of routing protocol that best fits the network at hand depends on its topology. [59] explores routing protocols in underwater acoustic networks. It presents an array of routing protocols that others have designed and that have made at least one of the following assumptions about its features.

- Clocks run in sync and without drift

- Static network

- Specialised hardware

- Location or geographical information known

- Predefined network settings

- Predefined network architecture

- Vertical communication only

- Reliable MAC dependent

- Sink node, to which all data flows, exists, is static and known

In contrast, the protocol in [59] makes none of them, as it strives to be a universal solution. Not only that, but it should also be able to handle long propagation delay well. The routing procedure starts with a node broadcasting a routing request. The packet header has an ID that blocks arrivals after the first from affecting the routing tables. When the routing request reaches the target node, it unicasts a response to the node that is associated with the first arrival to backtrack the route. Routes are maintained by data packets in that the receiver acknowledges received packets. If the receiver fails to deliver an acknowledgement in any form back to the sender, the route is considered broken and a new route is formed. The protocol is reactive.

[17] presents two geographical routing strategies that intend to solve the data gathering problem. Both delay-tolerant and delay-sensitive networks are considered. These strategies work in static network topologies, and their experiments do not appear to use any mobile nodes. The modems transmit packets back-to-back, not letting go of the channel until the packets are all 
sent. The routing algorithms are based on solving two similar optimisation problems, where the delay-sensitive problem has extra constraints. The interested reader may refer to [17, §§5-6] for further details.

[60] proposes a cross-layer protocol for adaptive routing. The nodes acquire information about the network by a first phase of exchanging control messages, then begin routing packets in the network. When routing, the proposed protocol, which is called NADIR, uses link quality, remaining energy of neighbours, energy needed and estimated hop count to select the next hop. The factors are normalised and weighted before the decision is made. The protocol is reactive.

[61] proposes an energy-aware routing protocol. It relies on "hello" messages to keep track of neighbours and computes an eligibility value from the residual energy and distance information. These factors are clumped together in a convex combination. The protocol is reactive.

[62] considers AUV networks in which the AUVs follow a predictable path. The routing solution used here is based on regions of uncertainty. Each AUV node estimates its own region, which is a cylinder, and shares the estimate with other AUVs. When a node wants to transmit data to a distant node, it uses local information to choose the neighbour that it thinks is the closest to the destination.

[39] presents a software-defined architecture that uses network function virtualisation to implement some of the OSI layers, including the network layer. Routing functionality is embedded in the control plane. This architecture, which is called SoftWater, has enabled several routing solutions. First, the network controllers can run routing optimisation algorithms. Second, SoftWater supports reactive strategies for handling node failure. Third, routes can be proactively redefined when mobile nodes, such as AUVs, are about to relocate.

[63] proposes a fusion between the network layer and data link layer. The proposed cross-layer model has only three layers, where its network layer contains both aforementioned layers.

[64] presents a class of energy-efficient routing strategies. Specifically, the energy required to reach a distant node with respect to the hop count is analysed, and using shorter and more hops is more efficient than using longer and fewer hops. There is also an optimal hopping distance. An array of hop destination selection strategies are presented. Most of them are distributed and can be implemented in practice. Some of them base their choice on a cone that faces the destination, some of them use circles and/or lines, one always chooses the nearest node that lies in the aforementioned cone, and the remainder are based on the shortest path and are there for reference only. This solution is geographical since the nodes know the network topology. 
Unet agent. [71] uses UnetStack agents to implement a TDMA MAC protocol, based on the work in [54], and tested it at sea.

\section{A. Cognitive acoustics}

An underwater network is not alone in general, but shares the medium with marine mammals or other underwater networks. This means some frequency bands might be occupied when a new network is deployed. Few networks consider this possibility, but there exists a variant of acoustic communication that does. Cognitive acoustics, as presented in [72], lets underwater acoustic nodes sense the channel for other acoustically communicating entities and adapt themselves to what they hear.

[72] investigates the challenges with cognitive acoustics. Most modems use frequency bands that overlap with those that sonars and marine mammals use, and concerns have been raised regarding the impact of anthropogenic acoustic noise on marine life. The spectrum can be underutilised in time and space. Temporal underutilisation occurs when nodes having low transmission duty cycle or mobile nodes using a frequency band. Spatial underutilisation occurs when one subspace of a network experiences more traffic than the rest of the network. Cognitive acoustics can be used to better utilise the spectrum by having it sense where the spectrum is used more and where it is used less. It consists of three elements. Spectrum sensing is the listening part of cognitive acoustics, which can be based on energy detection, power spectral density (PSD) estimation, waveforms or cyclostationarity. Spectrum management is a MAC protocol that consists of three blocks: sensing strategy, decision and sharing. Dynamic power control is the third part of cognitive acoustics.

The challenges with cognitive acoustics are plenty. First, spectrum sensing must handle attenuation and silently listening marine mammals. Second, the channel is highly variable, so the cognitive radio approach to dynamic power control will not extend to cognitive acoustics. Third, the sensing strategy needs to be considered from a practical perspective. Fourth, the decision method is prone to false alarms and missed transmissions due to propagation delay. Fifth and last, sharing resources is difficult due to the exposed terminal problem and limited bandwidth.

\section{Conclusion}

Underwater communication has become more interesting to industry and research groups around the world, whether sound, light or magnetic induction is used. This is thanks to the wide 
array of applications that underwater networking has in both industry and academia.

We have reviewed and presented a variety of advancements in underwater acoustic networking. Many of the research challenges that are presented in [7] have been addressed to a varying extent, although not all of them apply to all types of underwater networks. Researchers have called for a paradigm shift towards software-defined, open-architecture modems to accelerate research on IoUT. Modem manufacturers have introduced software-defined functionality in their products. At the same time, acoustic modems are still quite expensive (with a few exceptions), so there is still the need for designing more economic modems without giving up too much performance.

Standardisation and standardised testing methods will play an important role in the development and comparison of novel networking protocols, adaptive algorithms and MAC strategies.

\section{Acknowledgements}

This project received funds from the Research Council of Norway, project number 302435, "Autonomous Underwater Fleets: from AUVs to AUFs through adaptive communication and cooperation schemes".

\section{References}

[1] Robert McClure, Tim Acker, and James Dawson. Environmental assessment and monitoring of ocean energy sites — a rapid, proven, and economical approach. In OCEANS 2010 MTS/IEEE SEATTLE, pages 1-5, 2010.

[2] Serena Parton, Fraser Dalgleish, Pierre-Philippe Beaujean, Bing Ouyang, and Frank Caimi. Acquisition and registration of bathymetric acoustic data and mofsli (multiple overlapping field of view serial laser imager). In 2013 MTS/IEEE OCEANS - Bergen, pages 1-8, 2013.

[3] Arijit Khan and Lawrence Jenkins. Undersea wireless sensor network for ocean pollution prevention. In $20083 r d$ International Conference on Communication Systems Software and Middleware and Workshops (COMSWARE '08), pages 2-8, 2008.

[4] Gianni Cario, Alessandro Casavola, Petrika Gjanci, Marco Lupia, Chiara Petrioli, and Daniele Spaccini. Long lasting underwater wireless sensors network for water quality monitoring in fish farms. In OCEANS 2017 - Aberdeen, pages 1-6, 2017.

[5] Mari Carmen Domingo. An overview of the internet of underwater things. Journal of Network and Computer Applications, 35(6):1879-1890, 2012.

[6] Kongsberg Maritime. Autonomous underwater vehicle, hugin. Online. Accessed 2021-10-19.

[7] Ian F. Akyildiz, Dario Pompili, and Tommaso Melodia. Underwater acoustic sensor networks: research challenges. Ad Hoc Networks, 3(3):257-279, 2005.

[8] Mohammed Jouhari, Khalil Ibrahimi, Hamidou Tembine, and Jalel Ben-Othman. Underwater wireless sensor networks: A survey on enabling technologies, localization protocols, and internet of underwater things. IEEE Access, 7:96879-96899, 2019. 
[9] D.B. Kilfoyle and A.B. Baggeroer. The state of the art in underwater acoustic telemetry. IEEE Journal of Oceanic Engineering, 25(1):4-27, 2000.

[10] Andrea Goldsmith. Wireless Communications. Cambridge University Press, 2005. $8^{\text {th }}$ printing.

[11] Paul A. van Walree. Propagation and scattering effects in underwater acoustic communication channels. IEEE Journal of Oceanic Engineering, 38(4):614-631, 2013.

[12] Martin Siderius, Michael B. Porter, Paul Hursky, and Vincent McDonald. Effects of ocean thermocline variability on noncoherent underwater acoustic communications. The Journal of the Acoustical Society of America, 121(4):1895-1908, 2007.

[13] Andreja Radosevic, John G. Proakis, and Milica Stojanovic. Statistical characterization and capacity of shallow water acoustic channels. In OCEANS 2009-EUROPE, pages 1-8, 2009.

[14] Brian Borowski. Characterization of a very shallow water acoustic communication channel. In OCEANS 2009, pages $1-10,2009$.

[15] Xueyuan Zhao, Zhuoran Qi, and Dario Pompili. Configuration learning in underwater optical links, 2020.

[16] Raffaele Guida, Emrecan Demirors, Neil Dave, and Tommaso Melodia. Underwater ultrasonic wireless power transfer:a battery-less platform for the internet of underwater things. IEEE Transactions on Mobile Computing, pages 1-1, 2020.

[17] Dario Pompili, Tommaso Melodia, and Ian F. Akyildiz. Distributed routing algorithms for underwater acoustic sensor networks. IEEE Transactions on Wireless Communications, 9(9):2934-2944, 2010.

[18] L. M. Brekhovskikh and Yu. P. Lysanov. Fundamentals of Ocean Acoustics. New York, 2003. 3rd edition.

[19] Michael B. Porter. The BELLHOP Manual and User's Guide: PRELIMINARY DRAFT. Heat, Light, and Sound Research, Inc., La Jolla, California, USA, 2011. [Online] Accessed 2021-08-09.

[20] Parastoo Qarabaqi and Milica Stojanovic. Statistical characterization and computationally efficient modeling of a class of underwater acoustic communication channels. IEEE Journal of Oceanic Engineering, 38(4):701-717, 2013.

[21] Bas Binnerts, Koen Blom, and Stefania Giodini. Analysis of underwater acoustic propagation in a harbour environment and its effect on communication. In OCEANS 2017 - Aberdeen, pages 1-6, 2017.

[22] L. Freitag, M. Grund, S. Singh, J. Partan, P. Koski, and K. Ball. The whoi micro-modem: an acoustic communications and navigation system for multiple platforms. In Proceedings of OCEANS 2005 MTS/IEEE, pages 1086-1092 Vol. 2, 2005.

[23] Giovanni Toso, Riccardo Masiero, Paolo Casari, Oleksiy Kebkal, Maksym Komar, and Michele Zorzi. Field experiments for dynamic source routing: S2c evologics modems run the sun protocol using the desert underwater libraries. In 2012 Oceans, pages 1-10, 2012.

[24] Dale Green and Steven McManus. Smart modems - underwater capabilities beyond communications. In 2012 Oceans Yeosu, pages 1-5, 2012.

[25] X. Yu. Wireline quality underwater wireless communication using high speed acoustic modems. In OCEANS 2000 MTS/IEEE Conference and Exhibition. Conference Proceedings (Cat. No.00CH37158), volume 1, pages 417-422 vol.1, 2000.

[26] Paul van Walree, Roald Otnes, and Trond Jenserud. Watermark: A realistic benchmark for underwater acoustic modems. In 2016 IEEE Third Underwater Communications and Networking Conference (UComms), pages 1-4, 2016.

[27] Mandar Chitre, Shiraz Shahabudeen, and Milica Stojanovic. Underwater acoustic communications and networking: Recent advances and future challenges. Marine Technology Society Journal, 42(1):103-116, 2008.

[28] Roberto Petroccia, João Alves, and Giovanni Zappa. Janus-based services for operationally relevant underwater applications. IEEE Journal of Oceanic Engineering, 42(4):994-1006, 2017.

[29] J. Potter, J. Alves, D. Green, G. Zappa, I. Nissen, and K. McCoy. The janus underwater communications standard. 2014 Underwater Communications and Networking (UComms), pages 1-4, 2014. 
[30] Janus wiki. [Online]. Accessed June 2021.

[31] Andrew Smerdon, Francisco Bustamante, and Michael Baker. The swigacoustic standard: An acoustic communication standard for the offshore energy community. In 2016 IEEE Third Underwater Communications and Networking Conference (UComms), pages 1-4, 2016.

[32] Henry Dol, Koen Blom, and Ernest van der Spek. Experiences with janus and efforts towards a common heavyduty underwater communication stack. In 2016 IEEE Third Underwater Communications and Networking Conference (UComms), pages 1-5, 2016.

[33] Bridget Benson, Ying Li, Brian Faunce, Kenneth Domond, Don Kimball, Curt Schurgers, and Ryan Kastner. Design of a low-cost underwater acoustic modem. IEEE Embedded Systems Letters, 2(3):58-61, 2010.

[34] Sandra Sendra, Jaime Lloret, Jose Miguel Jimenez, and Lorena Parra. Underwater acoustic modems. IEEE Sensors Journal, 16(11):4063-4071, 2016.

[35] Jeff Neasham. About usmart. Online. Accessed 2021-10-28.

[36] Nils Morozs, Paul D. Mitchell, Yuriy Zakharov, Rahul Mourya, Yvan R. Petillot, Tyler Gibney, Mauro Dragone, Benjamin Sherlock, Jeffrey A. Neasham, Charalampos C. Tsimenidis, Mohammed E. Sayed, Alistair C. McConnell, Simona Aracri, and Adam A. Stokes. Robust tda-mac for practical underwater sensor network deployment: Lessons from usmart sea trials. In Proceedings of the Thirteenth ACM International Conference on Underwater Networks \& Systems, WUWNet '18, New York, NY, USA, 2018. Association for Computing Machinery.

[37] Yung-Ting Hsieh, Mehdi Rahmati, and Dario Pompili. Fd-uwa: Full-duplex underwater acoustic comms via selfinterference cancellation in space. In 2020 IEEE 17th International Conference on Mobile Ad Hoc and Sensor Systems (MASS), pages 256-264, 2020.

[38] Emrecan Demirors, George Sklivanitis, G. Enrico Santagati, Tommaso Melodia, and Stella N. Batalama. A high-rate software-defined underwater acoustic modem with real-time adaptation capabilities. IEEE Access, 6:18602-18615, 2018.

[39] Ian F. Akyildiz, Pu Wang, and Shih-Chun Lin. Softwater: Software-defined networking for next-generation underwater communication systems. Ad Hoc Networks, 46:1-11, 2016.

[40] Emrecan Demirors, Jiacheng Shi, Anh Duong, Neil Dave, Raffaele Guida, Bernard Herrera, Flavius Pop, Guofeng Chen, Cristian Casella, Sayedamirhossein Tadayon, Matteo Rinaldi, Stefano Basagni, Milica Stojanovic, and Tommaso Melodia. The seanet project: Toward a programmable internet of underwater things. In 2018 Fourth Underwater Communications and Networking Conference (UComms), pages 1-5, 2018.

[41] Henry S. Dol, Paolo Casari, Timo van der Zwan, and Roald Otnes. Software-defined underwater acoustic modems: Historical review and the nilus approach. IEEE Journal of Oceanic Engineering, 42(3):722-737, 2017.

[42] John Potter, João Alves, Thomas Furfaro, Arjan Vermeij, Nicolas Jourden, Diego Merani, Giovanni Zappa, and Alessandro Berni. Software defined open architecture modem development at cmre. In 2014 Underwater Communications and Networking (UComms), pages 1-4, 2014.

[43] Ieee standard for information technology-telecommunications and information exchange between systems - local and metropolitan area networks-specific requirements - part 11: Wireless lan medium access control (mac) and physical layer (phy) specifications. IEEE Std 802.11-2020 (Revision of IEEE Std 802.11-2016), pages 1-4379, 2021.

[44] Konstantinos Pelekanakis, Luca Cazzanti, Giovanni Zappa, and João Alves. Decision tree-based adaptive modulation for underwater acoustic communications. In 2016 IEEE Third Underwater Communications and Networking Conference (UComms), pages 1-5, 2016.

[45] Amir Tadayon and Milica Stojanovic. Iterative sparse channel estimation and spatial correlation learning for multichannel acoustic ofdm systems. IEEE Journal of Oceanic Engineering, 44(4):820-836, 2019. 
[46] Konstantinos Pelekanakis and Mandar Chitre. A channel-estimate-based decision feedback equalizer robust under impulsive noise. In 2014 Underwater Communications and Networking (UComms), pages 1-5, 2014.

[47] Henry Dol, Mathieu Colin, Paul van Walree, and Roald Otnes. Field experiments with a dual-frequency-band underwater acoustic network. In 2018 Fourth Underwater Communications and Networking Conference (UComms), pages 1-5, 2018.

[48] Christian R. Berger, Shengli Zhou, James C. Preisig, and Peter Willett. Sparse channel estimation for multicarrier underwater acoustic communication: From subspace methods to compressed sensing. IEEE Transactions on Signal Processing, 58(3):1708-1721, 2010.

[49] Prasad Anjangi and Mandar Chitre. Model-based data-driven learning algorithm for tuning an underwater acoustic link. In 2018 Fourth Underwater Communications and Networking Conference (UComms), pages 1-5, 2018.

[50] Donald A. Berry and Bert Fristedt. Bandit Problems: Sequential Allocation of Experiments. Monographs on Statistics and Applied Probability. Springer, Dordrecht, 1985.

[51] Paul van Walree, Helge Buen, and Roald Otnes. A performance comparison between dsss, m-fsk, and frequency-division multiplexing in underwater acoustic channels. In 2014 Underwater Communications and Networking (UComms), pages $1-5,2014$.

[52] M. Stojanovic, J.A. Catipovic, and J.G. Proakis. Phase-coherent digital communications for underwater acoustic channels. IEEE Journal of Oceanic Engineering, 19(1):100-111, 1994.

[53] Dario Pompili and Ian F. Akyildiz. Overview of networking protocols for underwater wireless communications. IEEE Communications Magazine, 47(1):97-102, 2009.

[54] Mandar Chitre, Mehul Motani, and Shiraz Shahabudeen. Throughput of networks with large propagation delays. IEEE Journal of Oceanic Engineering, 37(4):645-658, 2012.

[55] Borja Peleato and Milica Stojanovic. Distance aware collision avoidance protocol for ad-hoc underwater acoustic sensor networks. IEEE Communications Letters, 11(12):1025-1027, 2007.

[56] L. Freitag, M. Stojanovic, S. Singh, and M. Johnson. Analysis of channel effects on direct-sequence and frequency-hopped spread-spectrum acoustic communication. IEEE Journal of Oceanic Engineering, 26(4):586-593, 2001.

[57] M. Stojanovic. Optimization of a data link protocol for an underwater acoustic channel. In Europe Oceans 2005, volume 1, pages 68-73 Vol. 1, 2005.

[58] Zhangyu Guan, Hovannes Kulhandjian, and Tommaso Melodia. Stochastic channel access in underwater networks with statistical interference modeling. IEEE Transactions on Mobile Computing, pages 1-1, 2020.

[59] Rony Hasinur Rahman, Craig Benson, and Michael Frater. Routing protocols for underwater ad hoc networks. In 2012 Oceans - Yeosu, pages 1-7, 2012.

[60] Roberto Petroccia, Konstantinos Pelekanakis, Joào Alves, Stefano Fioravanti, Stephane Blouin, and Sean Pecknold. An adaptive cross-layer routing protocol for underwater acoustic networks. In 2018 Fourth Underwater Communications and Networking Conference (UComms), pages 1-5, 2018.

[61] Yonca Bayrakdar. Energy aware underwater routing: A pool experiment. In 2017 25th Telecommunication Forum (TELFOR), pages 1-4, 2017.

[62] Baozhi Chen and Dario Pompili. A qos-aware underwater optimization framework for inter-vehicle communication using acoustic directional transducers. IEEE Transactions on Wireless Communications, 13(5):2490-2504, 2014.

[63] Yangze Dong and Hefeng Dong. Simulation study on cross-layer design for energy conservation in underwater acoustic networks. In 2013 OCEANS - San Diego, pages 1-5, 2013.

[64] Michele Zorzi, Paolo Casari, Nicola Baldo, and Albert F. Harris. Energy-efficient routing schemes for underwater acoustic networks. IEEE Journal on Selected Areas in Communications, 26(9):1754-1766, 2008. 
[65] Veronika Kebkal, Konstantin Kebkal, and Oleksiy Kebkal. Experiments with network coding in dynamic underwater acoustic channel. In 2014 Underwater Communications and Networking (UComms), pages 1-4, 2014.

[66] João Galante, Paulo Sousa Dias, Manuel Ribeiro, Himanshi Jain, P.B. Sujit, and João Borges de Sousa. Flying acoustic modem: Results and future concepts of operation. In 2018 Fourth Underwater Communications and Networking Conference (UComms), pages 1-4, 2018.

[67] P.B. Sujit, Joao Sousa, and F. L. Pereira. Uav and auvs coordination for ocean exploration. In OCEANS 2009-EUROPE, pages 1-7, 2009.

[68] Mandar Chitre, Rohit Bhatnagar, and Wee-Seng Soh. Unetstack: An agent-based software stack and simulator for underwater networks. In 2014 Oceans - St. John's, pages 1-10, 2014.

[69] Mandar Chitre. Underwater networks handbook. https://unetstack.net/handbook. Accessed 2021-07-21.

[70] Mandar Chitre, Rohit Bhatnagar, Manu Ignatius, and Shailabh Suman. Baseband signal processing with unetstack. In 2014 Underwater Communications and Networking (UComms), pages 1-4, 2014.

[71] Prasad Anjangi and Mandar Chitre. Experimental demonstration of super-tdma: A mac protocol exploiting large propagation delays in underwater acoustic networks. In 2016 IEEE Third Underwater Communications and Networking Conference (UComms), pages 1-5, 2016.

[72] Yu Luo, Lina Pu, Michael Zuba, Zheng Peng, and Jun-Hong Cui. Challenges and opportunities of underwater cognitive acoustic networks. IEEE Transactions on Emerging Topics in Computing, 2(2):198-211, 2014.

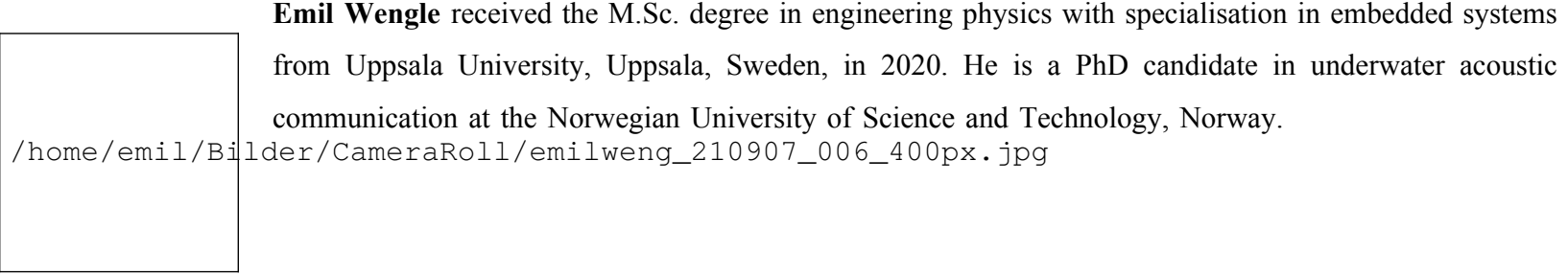


John Potter (SM'96) received joint honors BSc. degree in mathematics and physics from Bristol University, Bristol, U.K., in 1979 and the Ph.D. degree in glaciology and oceanography from the University of Cambridge, Cambridge, U.K., in 1985.

/home/emil/Hämtningar/potte-2695759-smal long He received the Polar Medal for this work by Queen Elizabeth II in 1988. He worked in Italy on propagation fluctuations in underwater acoustics from 1986 to 1991, then sailed across the Atlantic and through the Panama canal to San Diego where he worked at the UCSD Scripps Institution of Oceanography on Ambient Noise Imaging and Marine Mammal Acoustics. In 1995, he sailed on with his family from San Diego to Singapore, where he founded the Acoustic Research Laboratory (ARL) and helped create the Tropical Marine Science Institute. He headed the ARL and was an Associate Director of the TMSI for 12 years, working on passive acoustic imaging, marine mammal acoustics, distributed autonomous intelligent sensing, underwater communications networking, and cooperative behavior. In 2004-2005, he took a year "sabbatical" with his family to circumnavigate the Indian Ocean by sailboat on a sponsored voyage of research, public outreach, and education with the aim of improving the awareness and quality of our species' stewardship of the ocean ecosystem. After co-chairing OCEANS Singapore in 2006, he returned to Italy, first as a Consultant, then as a Project Leader and currently as the Principal Strategic Development Officer at the NATO STO Center for Maritime Research and Experimentation.

Dr. Potter has been an active member of the IEEE OES for more than 20 years, serving three terms on the Administrative Committee. He is also an Associate Editor for the IEEE Journal of Oceanic Engineering and an International Fellow of the Explorer's Club.

\footnotetext{
Hefeng Dong received the B.Sc. and M.Sc. degrees in physics from the Northeast Normal University, China in 1983 and 1986, respectively, and the Ph. D. degree in geoacoustics from the Jilin University, China, in 1994. she was associate professor from 1995 to 2000. She was visiting scholar and post doctorial fellow at the Norwegian University of Science and Technology, Norway between 1999 and 2001. From 2001 to 2002 she worked as a research scientist at the SINTEF Petroleum Research, Norway. Since 2002 she has been Professor in Acoustic Remote Sensing with the Norwegian University of Science and Technology, Norway. She was on sabbatical with the Underwater Acoustics Laboratory, University of Victoria, Canada, the College of Earth, Ocean and Environment, University of Delaware, USA, and the Laboratory of Mechanics and Acoustics, France in the periods of 2008-2009, 2014-2015, and 2019-2020, respectively. Her research interests include wave propagation, passive acoustics, geoacoustic modelling and inversion, signal processing in ocean acoustics and seismic, and underwater acoustic communication. Dr. Dong is a member of the Acoustical Society of America and IEEE.
} 